\title{
Ablação corneana baseada em topogirafia para correção das descentralizações pós-laser in situ keratomileusis: relato de três casos
}

\author{
Topographically supported customized ablation for the treatmentof decentered \\ laser in situ keratomileusis: report of three cases
}

\author{
Vinícius Coral Ghanem ${ }^{1}$ \\ Emir Amin Ghanem² \\ Denise Caon de Souza ${ }^{3}$ \\ Giselle Caon de Souza ${ }^{4}$ \\ Gustavo Campelo Bornhold $t^{5}$ \\ Milene Tiburcio Ferradoza ${ }^{6}$
}

\begin{tabular}{|c|}
\hline RESUMO \\
\hline Foram descritos três casos de pacientes submetidos à ablação corneana \\
baseada em topografia para a correção das descentralizações pós-laser in \\
situ keratomileusis, com sucesso pós-operatório.
\end{tabular}

Descritores: Ceratomileuse assistida por excimer laser in situ; Topografia da córnea; Astigmatismo; Relato de casos [tipo de publicação]

\section{INTRODUÇ̃̃O}

Apesar dos bons resultados obtidos com as técnicas do laser in situ keratomileusis (LASIK) e da ceratotomia foto-refrativa (PRK) para a correção dos erros refrativos, os astigmatismos irregulares secundários às ablações descentralizadas, aos traumas perfurantes do globo ocular e aos transplantes penetrantes de córnea são de difícil tratamento. Para a maioria das irregularidades corneanas, a cirurgia refrativa convencional não apresenta resultados satisfatórios. Além disso, a correção com óculos ou com com lentes de contato, muitas vezes, não é tolerada pelo paciente ou não corrige a acuidade visual satisfatoriamente ${ }^{(1-5)}$. Portanto, a ablação baseada em topografia torna-se uma alternativa para a correção do erro refrativo e regularização da superfície corneana nesses olhos.

Descrevemos aqui, o resultado da correção de 4 olhos de 3 pacientes que apresentavam ablações descentralizadas pós-LASIK, utilizando-se a ablação corneana baseada em topografia.

\section{RELATO DOS CASOS}

\section{Caso no 1: Descentralização pós-LASIK para correção miópica}

Paciente MRP, 32 anos, administradora, submetida à cirurgia para a correção de miopia há 5 meses, dos dois olhos, com a técnica do LASIK e insatisfeita com a visão. Refração pós-cirúrgica: $\mathrm{OD}-2,00 \mathrm{DE}-1,25 \mathrm{DC}$ x $65^{\circ} \mathrm{e}$ OE -0,75 DE -0,50 DC x 40 . A acuidade visual com correção (AVcc), em ambos os olhos (AO), era de 20/40. A topografia apresentava astigmatismo irregular devido a uma descentralização pós-LASIK em AO (Figura 1 e 2). O padrão de ablação foi calculado baseado na topografia utilizando-se o TOSCA (topographically supported customized ablation - sistema de ablação personalizada baseada em topografia), e está demonstrado nas figuras 3 e 4 . As topografias pós-operatórias (6 meses) mostravam maior regularidade corneana na 


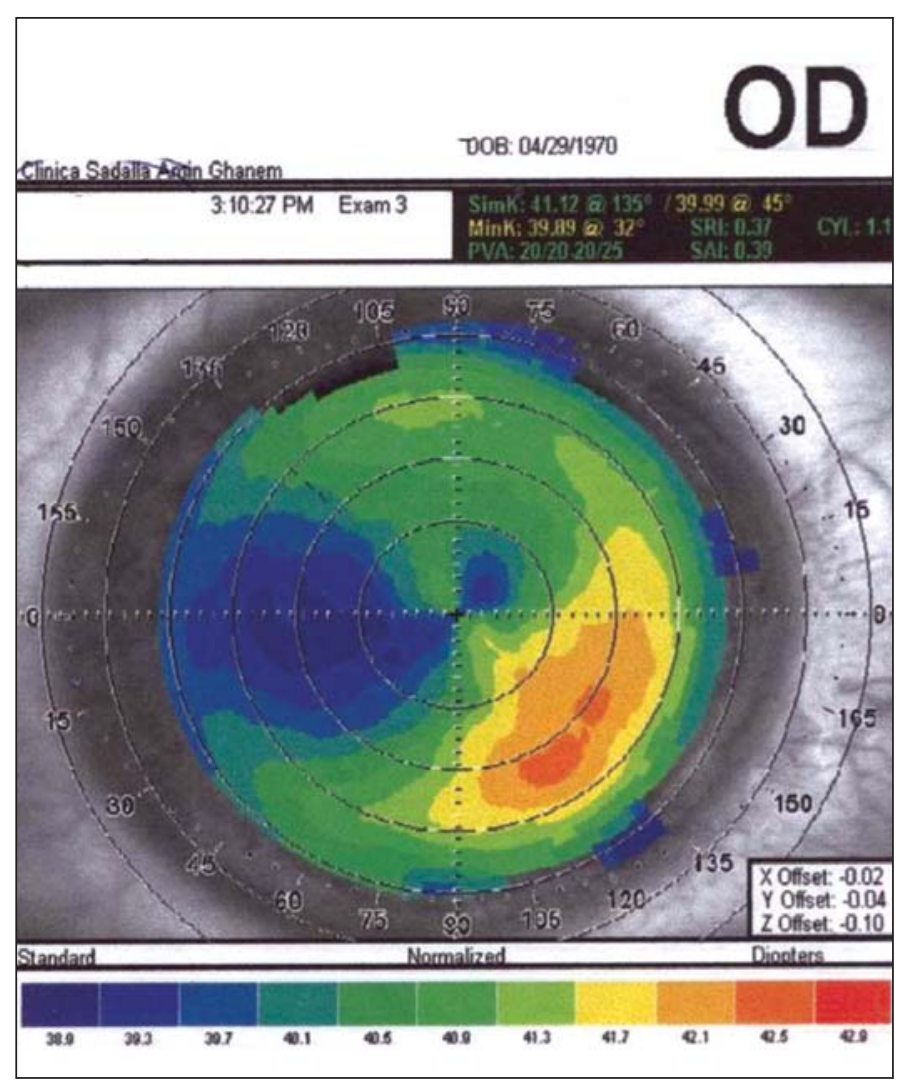

Figura 1 - Caso 1: Topografia pré-tosca do OD

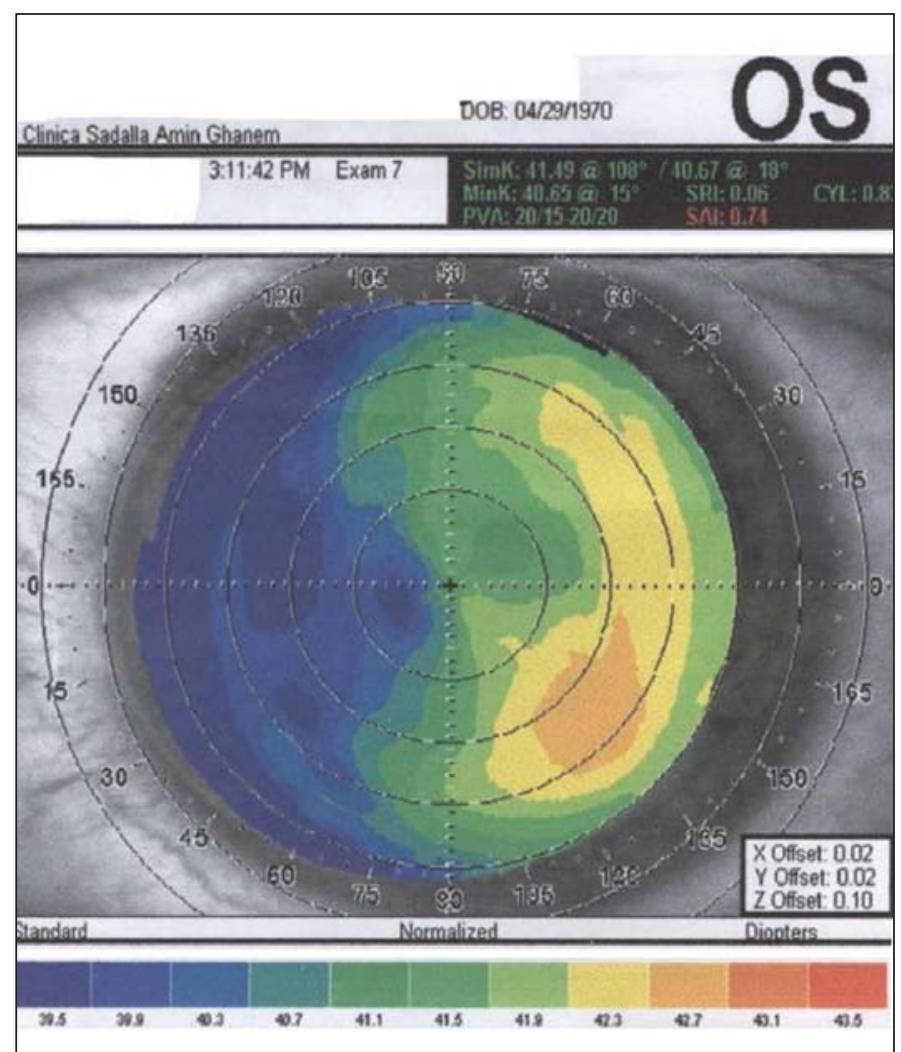

Figura 2 - Caso 1: Topografia pré-tosca do OE

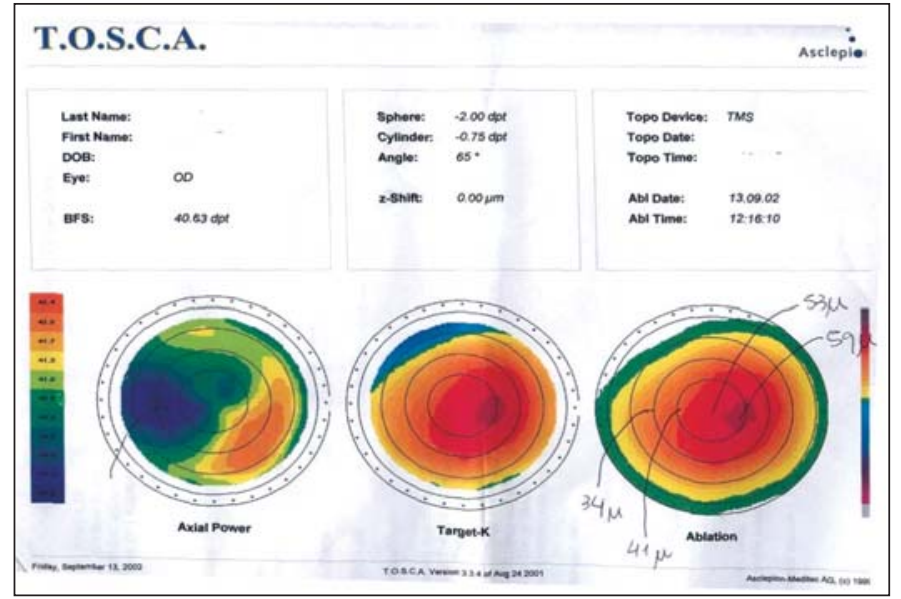

Figura 3 - Caso 1: Plano cirúrgico do OD

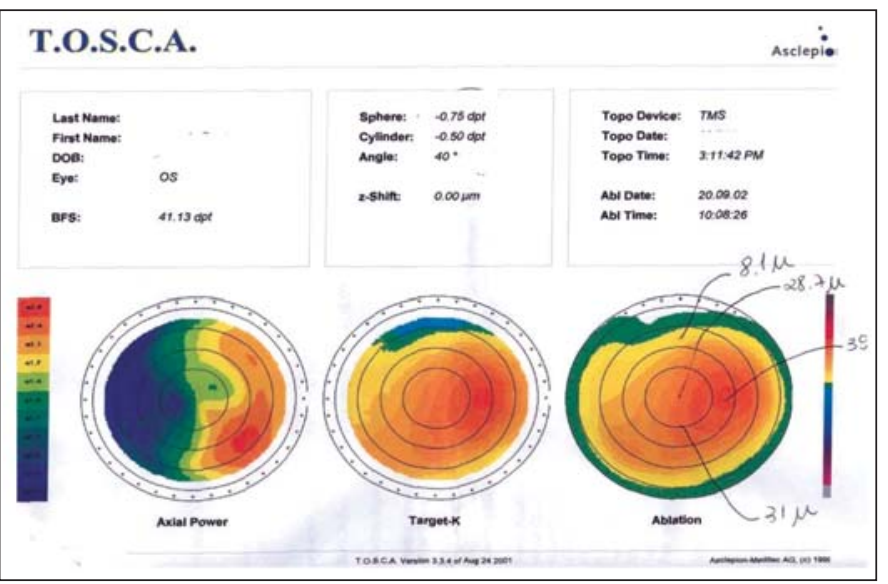

Figura 4 - Caso 1: Plano cirúrgico do $\mathrm{OE}$

zona óptica (Figuras 5 e 6). A AV sem correção (AVsc) em AO melhorou para 20/30 e a AVcc para 20/25 (Refração: OD -0,25 DE $-0,50 \mathrm{DC}$ x $80^{\circ}$; OE plano $-0,50 \mathrm{DC}$ x $80^{\circ}$ ).

\section{Caso $n^{\circ}$ 2: Descentralização pós-LASIK para correção miópica}

Paciente MS, 29 anos, farmacêutica. Foi submetida à cirurgia para a correção de miopia (-1,75 DE - 0,25 DC x $\left.5^{\circ}\right)$ do OD há 5 meses e queixava-se de visão borrada e cefaléia. A refração póscirúrgica era de +1,75 DE com AVcc de 20/30 e AVsc de 20/40. A topografia apresentava um astigmatismo irregular secundário a uma ablação descentralizada pós-LASIK (Figura 7). Após 4 meses da realização do TOSCA, a topografia mostrava menor irregularidade corneana na zona óptica (Figura 8). A AVsc era de 20/30 e a AVcc 20/25 (Refração: +1,00 DE-0,50 DC x 105).

\section{Caso $n^{\circ}$ 3: Descentralização pós-LASIK para correção hipermetrópica}

Paciente MSMT, 45 anos, costureira, submetida à cirurgia para a correção de hipermetropia (+2,50 DE -0,50 DC x 95²) do 


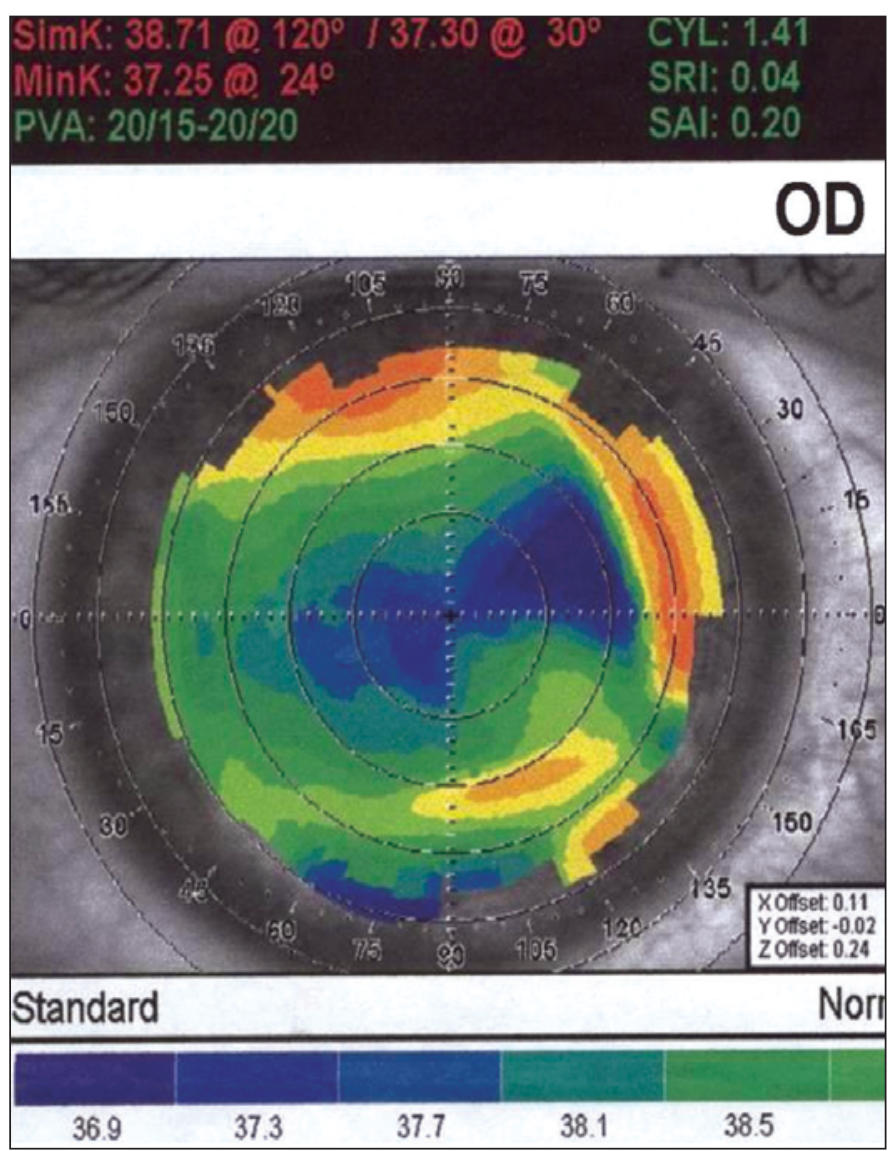

Figura 5 - Caso 1: Resultado pós-tosca do OD

OE há dois anos e referia baixa visual para longe. A refração pós-cirúrgica era $+1,50 \mathrm{DE}-2,00 \mathrm{DC} \times 80^{\circ} \mathrm{com} \mathrm{AV}$ cc de 20/50. A topografia pós-operatória mostrava ablação descentralizada (Figura 9). Após 4 meses da realização do TOSCA, a topografia evidenciava maior regularidade corneana na zona óptica (Figura 10). AVsc era de 20/40 e a AVcc 20/30 (Refração: +0,50 DE $-1,00$ DC $x 45^{\circ}$ ).

Os discos corneanos foram levantados para a realização do novo tratamento.

Todos os pacientes operados neste estudo ficaram muito satisfeitos com a cirurgia e referiram melhora significativa da qualidade visual.

\section{DISCUSSÃO}

Quarenta por cento das córneas humanas mostram alguma irregularidade que não pode ser corrigida com o tratamento cirúrgico convencional a laser( ${ }^{(6)}$.

Alguns pacientes apresentam-se desapontados com os resultados insatisfatórios produzidos pelo astigmatismo irregular pós-operatório resultante de um tratamento inadequado ${ }^{(7)}$.

Nassaralla e Nassaralla ${ }^{(8)}$ observaram que o sistema de "eye tracking" (sistema de radar do laser que evita a descentralização da ablação) é importante na correção de altos erros refrativos ou

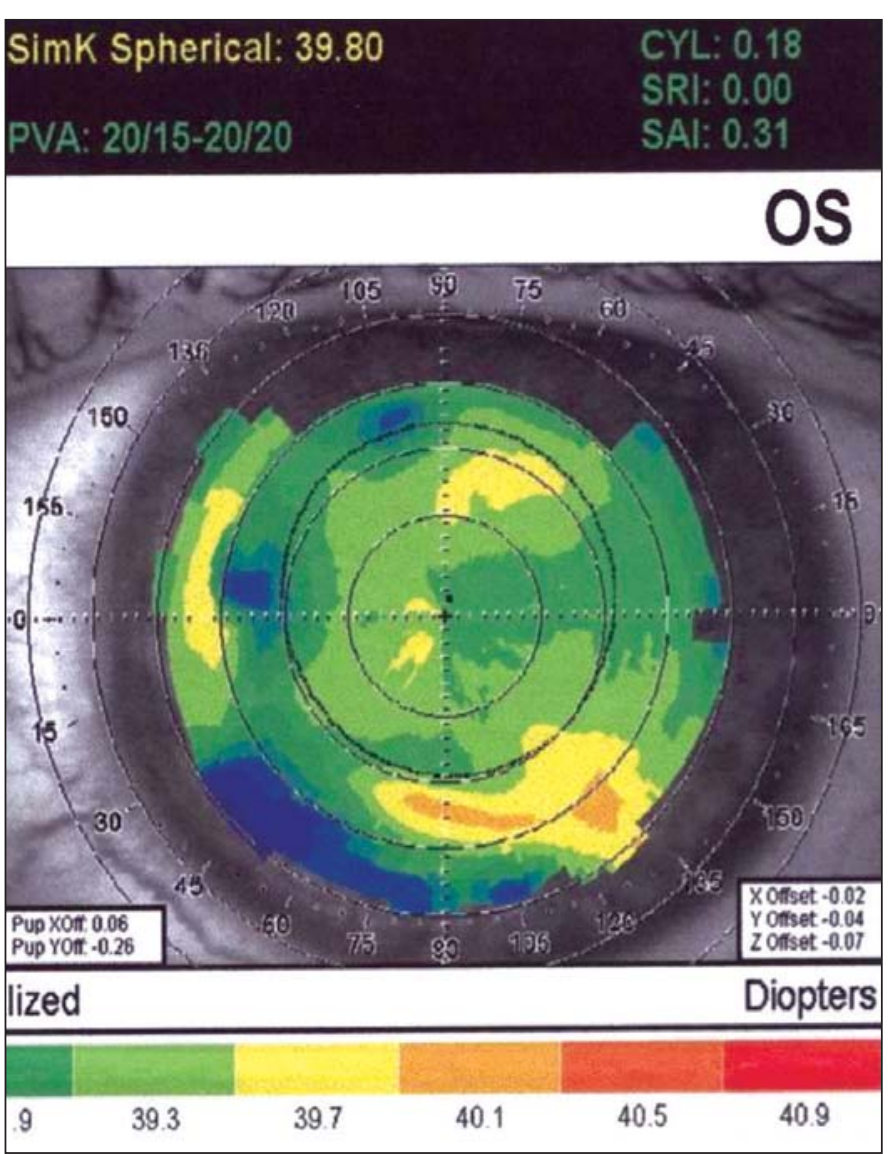

Figura 6 - Caso 1: Resultado pós-tosca do OE

quando a ablação leva um tempo maior para sua completa finalização, mas não elimina a ocorrência das descentralizações ${ }^{(9)}$.

Além do sistema "eye tracking", a experiência do cirurgião e a cooperação do paciente (como fixação, movimentos sacádicos e alinhamento da cabeça do paciente) contribuem para evitar as descentralizações ${ }^{(10)}$.

Uma ablação é dita excêntrica quando o seu centro não corresponde ao centro do eixo óptico. Isso é uma séria complicação, pois induz a formação de uma zona óptica multifocal criando problemas visuais ${ }^{(11-12)}$ como diplopia monocular e diminuição da sensibilidade ao contraste ${ }^{(13-16)}$.

Valores baixos de descentralização (de 0,5 a $1,00 \mathrm{~mm}$ ) poderiam afetar a $\mathrm{AV}$ de baixo contraste ${ }^{(17)} \mathrm{e}$ induzir a aberrações de alta ordem na análise da frente de onda ${ }^{(18)}$. Quando uma descentralização é significante (maior que 1,00 mm) ocorre um grande comprometimento da AV com sintomas visuais ${ }^{(19)}$.

$\mathrm{Na}$ tentativa de corrigir os astigmatismos irregulares, várias técnicas foram propostas. Alguns autores apresentaram dois casos de descentralização após PRK retratados com nova cirurgia de PRK onde um círculo de epitélio aderido sobre a área de ablação descentralizada serviu como uma máscara ${ }^{(20)}$. Outros autores descreveram uma técnica de retratamento com laser transepitelial utilizando metilcelulose para proteger as áreas mais profundas da zona de ablação descentralizada ${ }^{(21)}$. 


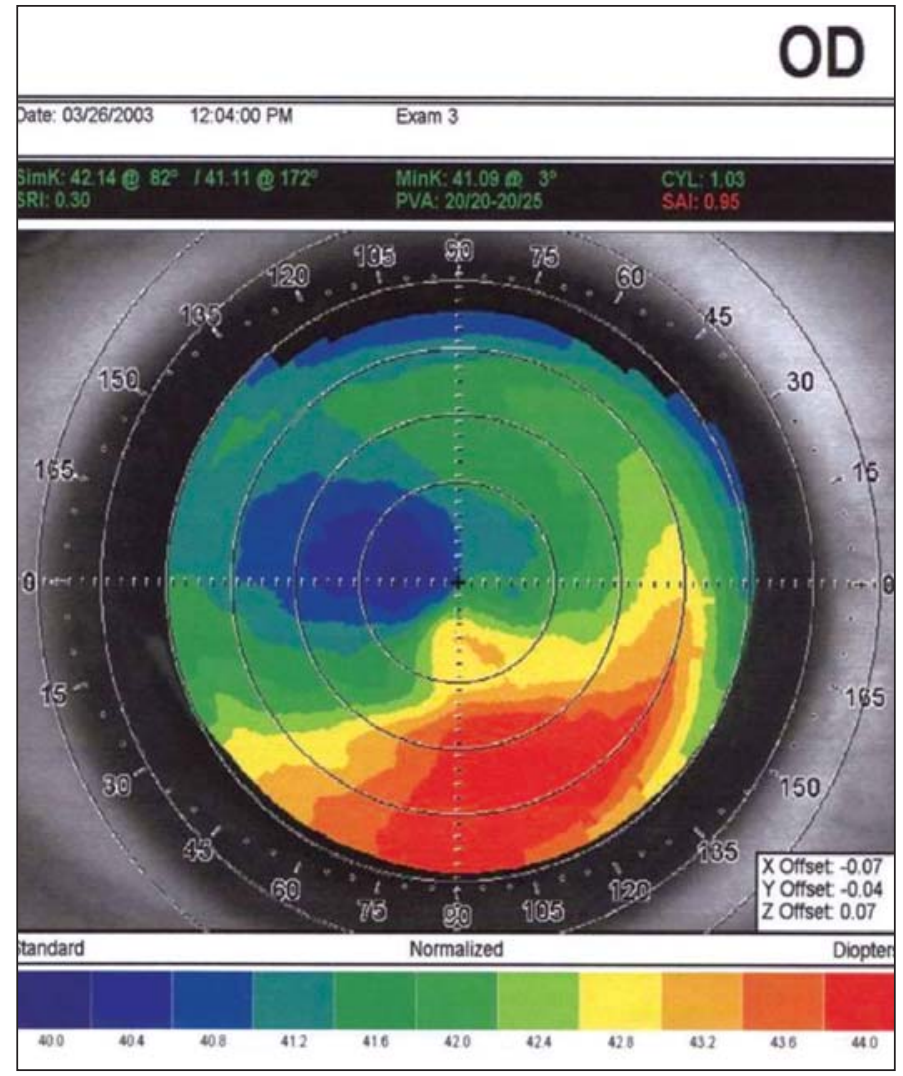

Figura 7 - Caso 2: Topografia pré-tosca do OD

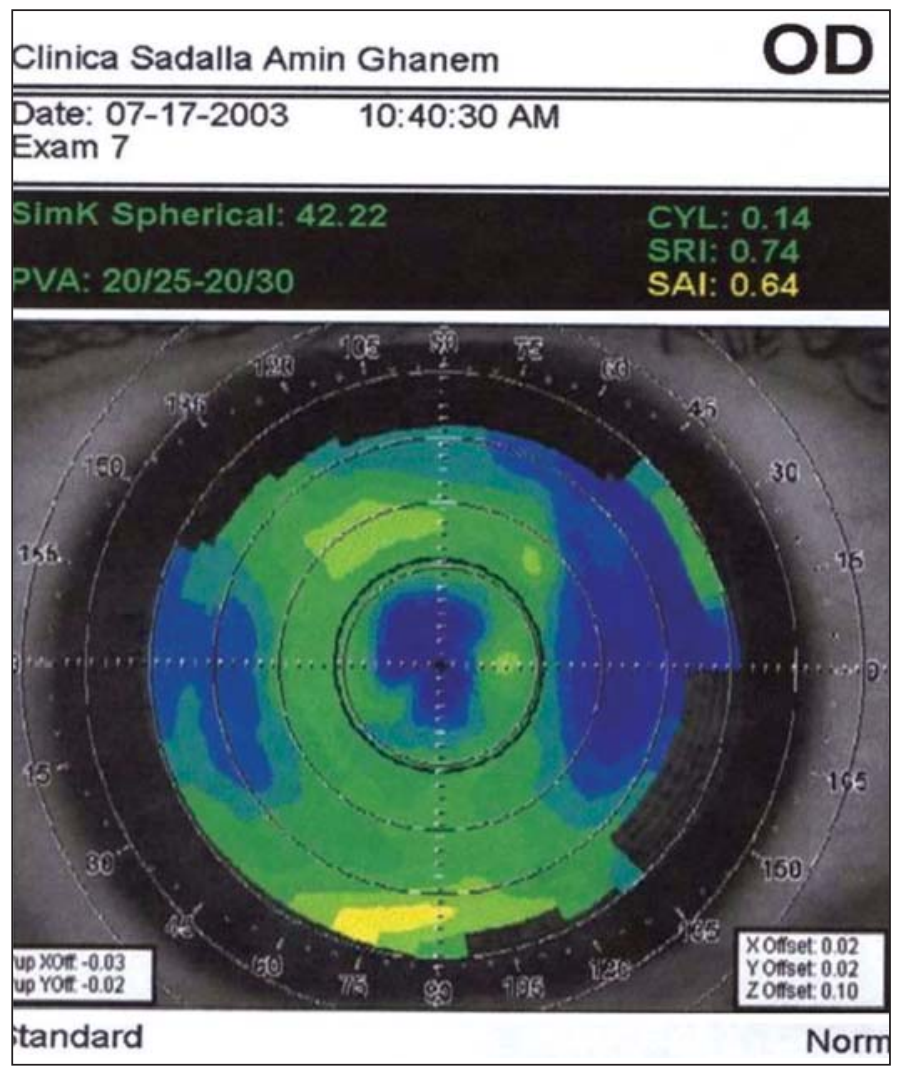

Figura 8 - Caso 2: Resultado pós-tosca do OD

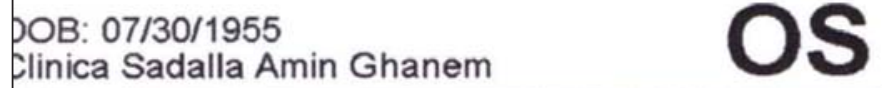

\begin{tabular}{|c|c|c|}
\hline $\begin{array}{l}\text { ate: } 09 / 06 / 2002 \\
\text { Exam } 7\end{array}$ & $5: 20: 46$ PM & \\
\hline $\begin{array}{l}\text { SimK: } 44.00 @ 177^{\circ} \\
\text { hinK: } 41.71 @ 93^{\circ} \\
\text { PVA: } 20 / 25-20 / 30\end{array}$ & $141.72 @ 87^{\circ}$ & $\begin{array}{l}\text { CYL: } 2.28 \\
\text { SRI: } 0.72 \\
\text { SAI: } 1.88\end{array}$ \\
\hline
\end{tabular}

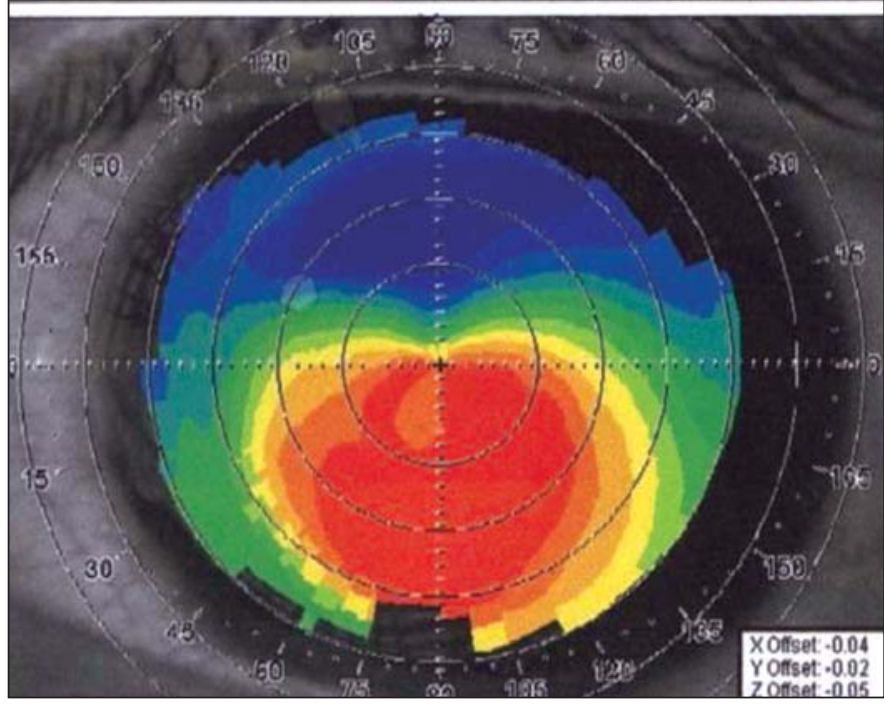

Figura 9 - Caso 3: Topografia pré-tosca do OE

DOB: $07 / 30 / 1955$

flinica Sadalla Amin Ghanem

OS

pate: 10/15/2002 3:03:36 PM

Exam 11

\begin{tabular}{ll}
\hline \hline $\operatorname{SimK}: 43.84 @ 163^{\circ} / 42.84 @ 73^{\circ}$ & CYL: 1.00 \\
hinK: 42.74 (i) $58^{\circ}$ & SRI: 0.67 \\
PVA: $20 / 20-20 / 30$ & SAl: 1.38
\end{tabular}

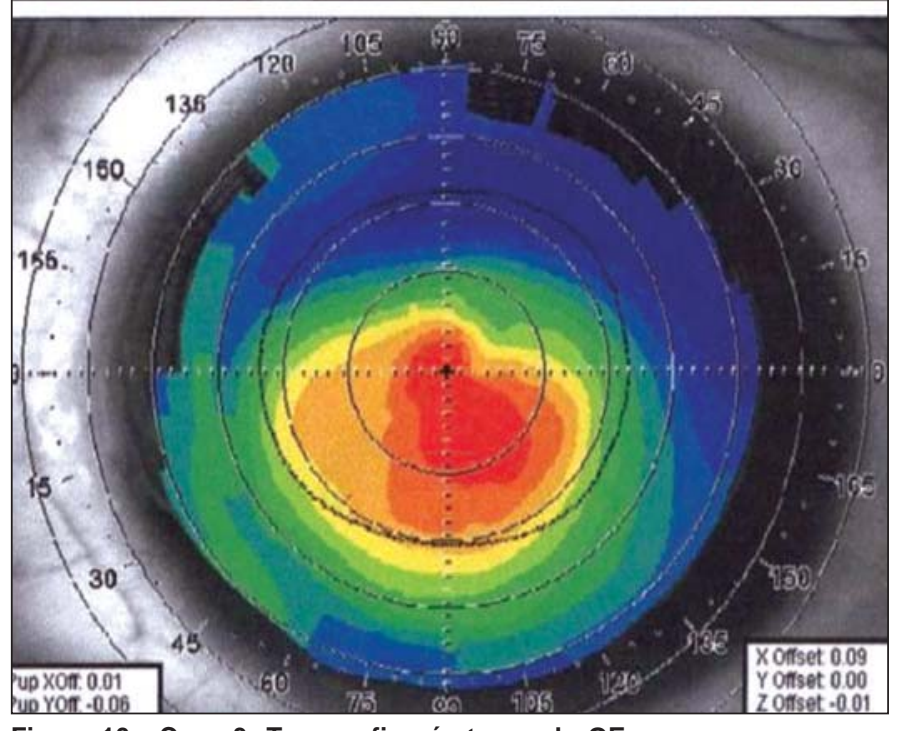

Figura 10 - Caso 3: Topografia pós-tosca do OE 
Alguns autores, em 1994, criaram um sistema de ablação baseado em topografia que consistia no tratamento focal, com ablações de diâmetros circulares pequenos, calculados com base no diâmetro e curvatura das áreas irregulares da superfície corneana ${ }^{(22)}$. Nos dois casos apresentados, houve redução do astigmatismo irregular e melhora da acuidade visual. Apesar das várias técnicas publicadas, muito ainda tinha que ser feito para se conseguir resultados mais satisfatórios.

Com o desenvolvimento de novos aparelhos de excimer laser, foi possível o emprego de técnicas para correção dos casos de irregularidade corneana, onde a análise topográfica é a base para o tratamento com o excimer laser. Um dos sistemas existentes para ablação personalizada da córnea, baseada na topografia, é conhecido como TOSCA (topography supported customized ablation - Meditec, Alemanha). Este sistema utiliza os mapas dos perfis de altura e de curvatura axial de cada córnea, analisados pelo aparelho Tomey TMS-2N ${ }^{\circledR}(\mathrm{Me}-$ ditec, Alemanha), e calcula o padrão de ablação necessário para transformar a superfície corneana numa superfície esférica dentro da zona óptica de 6 milímetros, sem alterar o equivalente esférico refracional, a menos que isso seja desejável. Esse aparelho fornece a topografia pré-operatória e o mapa topográfico que ilustra a ablação programada ponto a ponto. Os dados são, então, transferidos para o aparelho de excimer laser (Meditec MEL 70 G-scan ${ }^{\circledR}$ ) que é um "flying-spot" (excimer laser rastreável de feixe pequeno) com perfil de ablação Gaussiana (2 mm de diâmetro, $50 \mathrm{~Hz}$, duração do pulso de $15 \mathrm{~ns}$ e nível de energia na córnea de $200 \mathrm{~mJ} / \mathrm{cm}^{2}$ ) e possui controle permanente de fixação ocular pelo "eye-tracker".

Ablações personalizadas como o TOSCA têm sido efetivas para os tratamentos das irregularidades corneanas de etiologias variadas $^{(23-27)}$.

Wiesinger-Jendritza, em 1998, utilizando a técnica do LASIK associada à ablação corneana baseada em topografia, relatou que em $81,3 \%$ dos casos houve redução do astigmatismo irregular e em 19,4\% houve correção total. Apesar de muitos casos apresentarem hipocorreção ou regressão ao longo do acompanhamento, não houveram complicações que resultaram em redução significativa da $\mathrm{AV}$ (perda de 2 ou mais linhas de visão corrigida ${ }^{(28)}$. Outro estudo semelhante relatou importante redução do cilindro refrativo, aumento da regulariadade corneana e melhora significativa da AV sem correção em pacientes com altos graus de astigmatismo irregular préoperatórios $^{(7)}$. Resultados semelhantes aos mostrados neste estudo, no qual todos os pacientes melhoraram a AV e apresentaram maior regularidade corneana pós-operatória.

Assim como neste estudo, outros autores, em 2004, utilizando o TOSCA em 11 olhos, para corrigir ablações descentralizadas, obtiveram uma significativa melhora da AV com e sem correção, redução dos sintomas visuais subjetivos (halos, ofuscamento, imagens fantasmas e diplopia monocular) e das aberrações ópticas de alta ordem. Além disso, após análise topográfica, observou-se diminuição significativa das descentralizações. O acompanhamento foi de aproximadamente 9 meses e somente 1 olho manteve o erro refrativo do pré-operatório ${ }^{(12)}$.
Avaliando os casos aqui descritos, observa-se que essa técnica de ablação corneana baseada em topografia apresenta bons resultados no tratamento das descentralização pósLASIK miópico e hipermetrópico, entretanto estudos com maior número de casos e acompanhamento pós-operatório mais prolongado, são necessários para confirmar esses resultados. Além disto, a tecnologia de wavefront, a qual propicia um melhor detalhamento do sistema óptico ocular, provavelmente fornecerá resultados ainda mais precisos no futuro.

\section{ABSTRACT}

Three cases of decentered laser in situ keratomileusis were succefully treated with the topographically supported customized ablation.

Keywords: Keratomileusis, laser in situ; Corneal topography; Astigmatism; Case reports [publication type]

\section{REFERÊNCIAS}

1. Seitz B, Langenbucher A, Kus MM, Harrer M. Experimental correction of irregular corneal astigmatism using topography-based flying-spot-mode excimer laser photoablation. Am J Ophthalmol. 1998;125(2):252-6.

2. Cavanagh HD, Leveille AS. Extended-wear contact lenses in patients with corneal grafts and aphakia. Ophthalmology. 1982;89(6):643-50.

3. McCartney DL, Whitney CE, Stark WJ, Wong SK, Bernitsky DA. Refractive keratoplasty for disabling astigmatism after penetrating keratoplasty. Arch Ophthalmol. 1987;105(7):954-7.

4. Karabatsas CH, Cook SD, Figueiredo FC, Diamond JP, Easty DL. Surgical control of late postkeratoplasty astigmatism with or without the use of computerized video keratography: a prospective, randomized study. Ophthalmology. 1998;105(11):1999-2006.

5. Genvert GI, Cohen EJ, Arentsen JJ, Laibson PR. Fitting gas-permeable contact lenses after penetrating keratoplasty. Am J Ophthalmol. 1985;99(5):511-4.

6. Bogan SJ, Waring GO 3rd, Ibrahim O, Drews C, Curtis L. Classification of normal corneal topography based on computer-assisted videokeratography. Arch Ophthalmol. 1990;108(7):945-9.

7. Hall GW, Campion M, Sorenson CM, Monthofer S. Reduction of corneal astigmatism at cataract surgery. J Cataract Refract Surg. 1991;17(4):407-14. Comment in: J Cataract Refract Surg. 1991;17(6):861.

8. Nassaralla BR, Nassaralla JJ. Laser in situ keratomileusis after penetrating keratoplasty. J Refract Surg. 2000;16(4):431-7.

9. McDonald MB, Carr JD, Frantz JM, Kozarsky AM, Maguen E, Nesburn AB, et al. Laser in situ keratomileusis for myopia up to -11 diopters with up to -5 diopters of astigmatism with the summit autonomous LADARVision excimer laser system. Ophthalmology. 2001;108(2):309-16.

10. Tsai YY, Lin JM. Ablation centration after active eye-tracker-assisted photorefractive keratectomy and laser in situ keratomileusis. J Cataract Refract Surg. 2000;26(1):28-34.

11. Webber SK, McGhee CN, Bryce IG. Decentration of photorefractive keratectomy ablation zones after excimer laser surgery for myopia. J Cataract Refract Surg. 1996;22(3):299-303.

12. Kymionis GD, Panagopoulou SI, Aslanides IM, Plainis S, Astyrakakis N, Pallikaris IG. Topographically supported customized ablation for the management of decentered laser in situ keratomileusis. Am J Ophthalmol. 2004;137 (5):806-11.

13. Lin DT, Sutton HF, Berman M. Corneal topography following excimer photorefractive keratectomy for myopia. J Cataract Refract Surg. 1993;19 Suppl:149-54

14. Seiler T, McDonnell PJ. Excimer laser photorefractive keratectomy. Surv Ophthalmol. 1995;40(2):89-118. Comment in: Surv Ophthalmol. 1996;41(2):187-8.

15. McGhee CN, Bryce IG. Natural history of central topographic islands following excimer laser photorefractive keratectomy. J Cataract Refract Surg. 1996; 22(9):1151-8. Comment in: J Cataract Refract Surg. 1999;25(3):302-4. 
16. Castillo A, Romero F, Martin-Valverde JA, Diaz-Valle D, Toledano N, Sayagues O. Management and treatment of central steep islands after excimer laser photorefractive keratectomy. J Refract Surg. 1996;12(6):715-20.

17. Verdon W, Bullimore M, Maloney RK. Visual performance after photorefractive keratectomy. A prospective study. Arch Ophthalmol. 1996;114(12):1465-72.

18. Mrochen M, Kaemmerer M, Mierdel P, Seiler T. Increased higher-order optical aberrations after laser refractive surgery: a problem of subclinical decentration. $\mathrm{J}$ Cataract Refract Surg. 2001;27(3):362-9.

19. Doane JF, Cavanaugh TB, Durrie DS, Hassanein KM. Relation of visual symptoms to topographic ablation zone decentration after excimer laser photorefractive keratectomy. Ophthalmology. 1995;102(1):42-7. Comment in: Ophthalmology. 1995;102(9):1258-60.

20. Lim-Bon-Siong R, Williams JM, Steinert RF, Pepose JS. Retreatment of decentered excimer photorefractive keratectomy ablations. Am J Ophthalmol. 1997;123(1):122-4.

21. Talamo JH, Wagoner MD, Lee SY. Management of ablation decentration following excimer photorefractive keratectomy. Arch Ophthalmol. 1995 Jun;113(6):706-7.
22. Gibralter R, Trokel SL. Correction of irregular astigmatism with the excimer laser. Ophthalmology. 1994;101(7):1310-4; discussion 1314-5.

23. Alessio G, Boscia F, La Tegola MG, Sborgia C. Topography-driven excimer laser for the retreatment of decentralized myopic photorefractive keratectomy. Ophthalmology. 2001;108(9):1695-703.

24. Alessio G, Boscia F, La Tegola MG, Sborgia C. Corneal interactive programmed topographic ablation customized photorefractive keratectomy for correction of postkeratoplasty astigmatism. Ophthalmology. 2001;108(11):2029-37.

25. Knorz MC, Wiesinger B, Liermann A, Seiberth V, Liesenhoff H. Laser in situ keratomileusis for moderate and high myopia and myopic astigmatism. Ophthalmology. 1998;105(5):932-40.

26. Knorz MC. Broad-beam versus scanning-beam lasers for refractive surgery. Ophthalmic Pract. 1997;15:142-5.

27. Alessio G, Boscia F, La Tegola MG, Sborgia C. Topography-driven photorefractive keratectomy: results of corneal interactive programmed topographic ablation software. Ophthalmology. 2000;107(8):1578-87.

28. Wiesinger-Jendritza B, Knorz MC, Hugger P, Liermann A Laser in situ keratomileusis assisted by corneal topography. J Cataract Refract Surg. 1998; 24(2):166-74

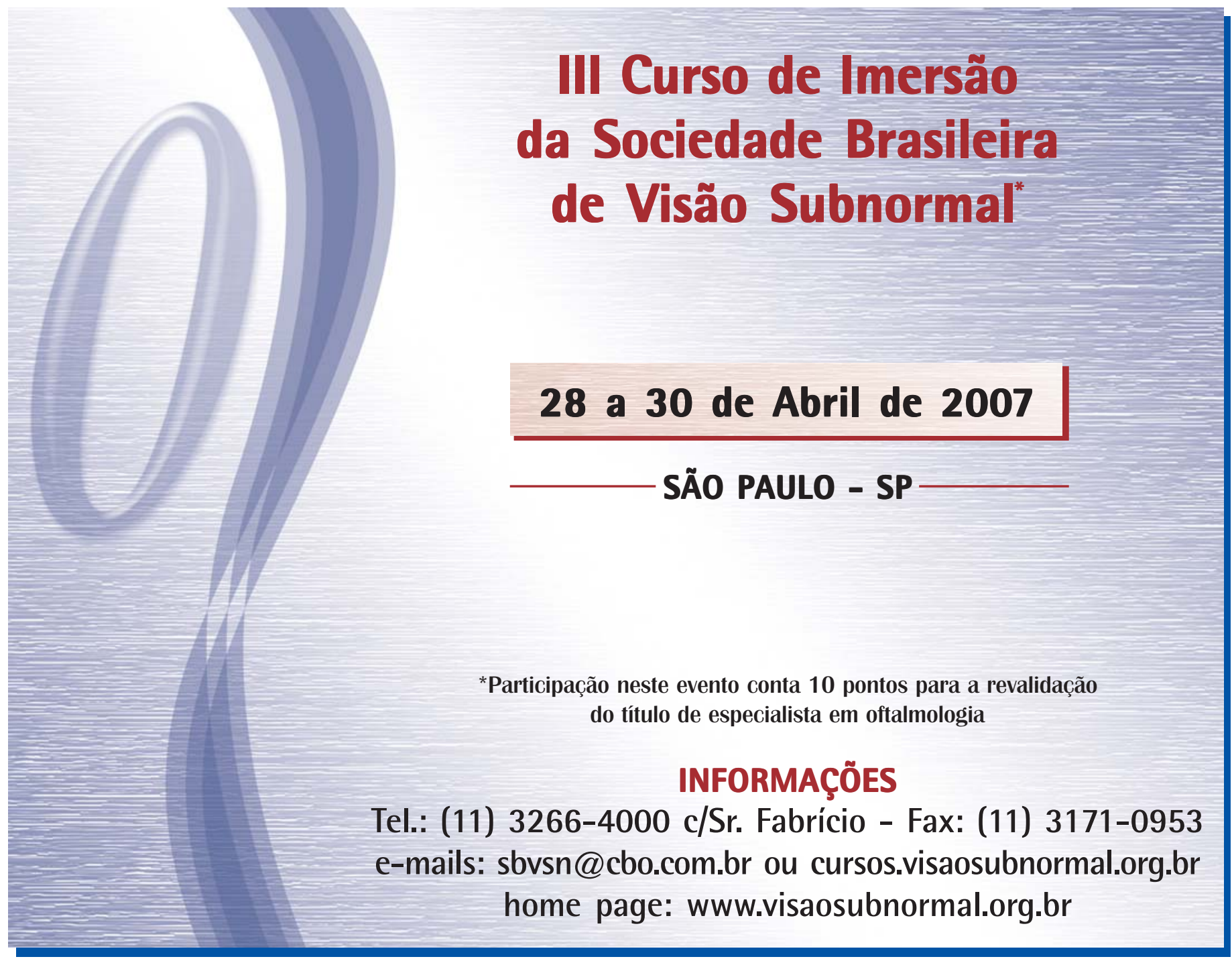

